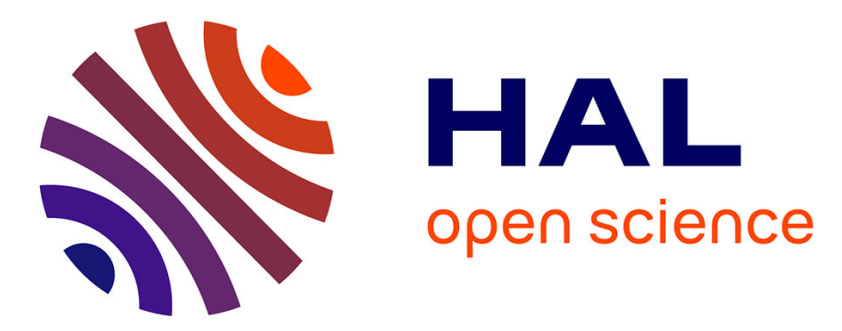

\title{
Smart gateways switching control algorithms based on tropospheric propagation forecasts
}

Nicolas Jeannin, Laurent Castanet, Isabelle Dahman, Vivien Pourret, Béatrice Pouponneau

\section{- To cite this version:}

Nicolas Jeannin, Laurent Castanet, Isabelle Dahman, Vivien Pourret, Béatrice Pouponneau. Smart gateways switching control algorithms based on tropospheric propagation forecasts. International Journal of Satellite Communications and Networking, 2017, page 1-13. 10.1002/sat.1233 . hal01735636

\section{HAL Id: hal-01735636 \\ https://hal.science/hal-01735636}

Submitted on 16 Mar 2018

HAL is a multi-disciplinary open access archive for the deposit and dissemination of scientific research documents, whether they are published or not. The documents may come from teaching and research institutions in France or abroad, or from public or private research centers.
L'archive ouverte pluridisciplinaire HAL, est destinée au dépôt et à la diffusion de documents scientifiques de niveau recherche, publiés ou non, émanant des établissements d'enseignement et de recherche français ou étrangers, des laboratoires publics ou privés. 


\title{
Smart Gateways Switching Control Algorithms Based on Tropospheric Propagation Forecasts
}

\author{
N. Jeannin ${ }^{1}$, L. Castanet ${ }^{1}$, I. Dahman ${ }^{1}$, V. Pourret ${ }^{2}$, B. Pouponneau ${ }^{2}$ \\ ${ }^{1}$ ONERA, DEMR, UFTMiP, \\ 2 Avenue Edouard Belin, 31055 Toulouse CEDEX 4,France \\ ${ }^{2}$ Météo France, DSM/EC/ECGC, \\ Avenue Gaspard Coriolis, 31000 Toulouse, France
}

\begin{abstract}
The use Q/V band spectrum for the feeder links of high throughput satellites and the need to cope with the significant propagation impairments at those frequencies motivate the development of smart diversity techniques. Those techniques aim at improving the availability level of the overall feeder link with a limited level of redundancy. The combinatorial gain of availability provided by those techniques can be obtained only if efficient switching methodologies are developed, performing the best trade-off between system flexibility and channel prediction accuracy.

This paper proposes various propagation forecast mechanisms for the control of switching between gateways in smart diversity, corresponding to various system assumptions in terms of required anticipation time for the triggering of the switches. The performances of those algorithms are then assessed against measured attenuation and meteorological data. It enables to evaluate the performance degradation with regards to an idealized case.
\end{abstract}

\section{Introduction}

The dramatic increase of bandwidth demand for high throughput telecommunication satellite systems fosters the development of feeder links at $\mathrm{Q} / \mathrm{V}$ and $\mathrm{W}$ band or also of optical feeder links. All those bands are particularly sensitive to propagation conditions. The power margins required to cope with fades in the atmosphere, especially in presence of cloud and rain, can reach several tens of decibels. For RF feeder links, even the use of all the spectrum available for satellite systems at those bands will require the use of a few tens of gateways to reach some hundreds of gigabits per second aggregated at system level [?], [?]. Therefore, the reduction in the power margins or in the feeder link unavailability by using classical site diversity is rather inefficient as it requires to double the already high number of gateways needed from a strict bandwidth perspective. Thus techniques using a limited number of redundant gateways shared by the nominal gateways of a system have been developed. They take advantage 
of the relatively low probability of simultaneous occurrence of large propagation impairments on different sites. Indeed, those propagation impairments are almost spatially uncorrelated if the distance between the various sites exceed some hundreds of kilometers. The use of those techniques can result in very low unavailability levels with a slight oversizing of the ground segment [?, ?, ?].

Those results have been established assuming that the handover between the nominal and backup gateways can be seamless and instantaneous. However in practice, the handovers will require an accurate prediction of the evolution of the propagation channel over a duration that can range from some seconds to some hours [?].

Depending on the accuracy of the propagation forecasts, on the latency of the switching process and on the temporary decrease in capacity caused by the traffic switching from one gateway to another, the system may experience operationally performance more or less significantly lower than the ones computed from a combinatorial analysis.It may be needed to take this degradation into account at design stage. In this paper an algorithm for the optimal control of switching between gateways taking into account those parameters is firstly developed. Then depending on the time horizon of the required propagation forecast, various rain attenuation forecast mechanisms are developed based on the instantaneous state of the channel, on weather radar data or on numerical weather forecasts. In a last part those solutions are evaluated using concurrent propagation measurements and meteorological forecasts to assess the performances of a network of gateways in operational conditions.

\section{System model}

In the following, a $N+P$ diversity system illustrated in figure 1 is considered. The $N$ nominal gateways $(G W)$ are each serving one of the $N$ groups of user beams $(U B G)$. If some of the gateway-satellite links undergo too severe propagation impairments the traffic may be re-routed through one of the $P$ redundant gateways.

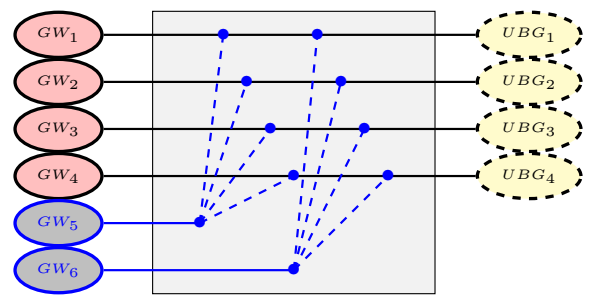

Figure 1: Principle of the $\mathrm{N}+\mathrm{P}$ diversity

A fraction $i / N$ of the system feeder link is in outage if $P+i$ gateways are simultaneously in outage. In order to avoid co-channel interferences, the gateways of the system need to be spaced by some hundreds of $\mathrm{km}$. Thus the propagation 


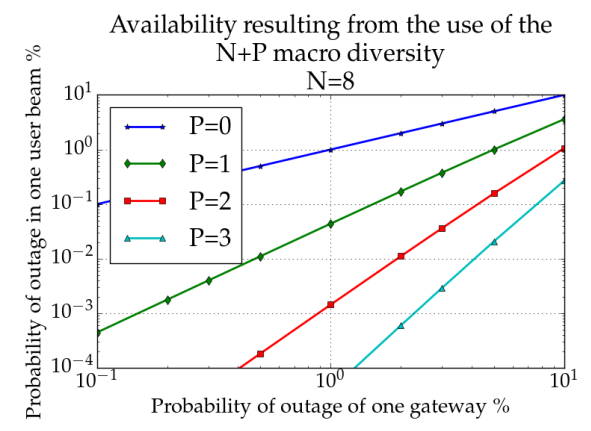

Figure 2: Overall capacity of the feeder link function of the outage probability of one gateway satellite-link

impairments on the various gateways can be considered as approximately uncorrelated. Assuming that each gateway has the same probability of unavailability $p$, the overall unavailability assuming the possibility of seamless handover of the system is given by Eq.1:

$$
u=\sum_{i=1}^{N} \frac{i}{N} \mathcal{C}_{N+P}^{P+i} p^{P+i}(1-p)^{N-i}
$$

As illustrated in figure 2, the inclusion of a limited number of redundant gateways results in a significant reduction of the unavailability for the whole feeder link.

\subsection{Modelling of smart diversity system}

In actual implementation of the diversity mechanisms the availability will differ from the combinatorial one evaluated with Eq. 1 for different reasons. First there will be some latency between two consecutive switches corresponding to the time to prepare re-routing of traffic from one gateway to another, the time to prepare the telecommand, and the time to activate the satellite on board switch. Then during the switch there may be a loss of link for a short duration and a capacity reduction. In addition, if all the terminals within a group of beams are losing the forward signal for a too long duration, the procedure to log again all the terminals into the service can be relatively long. One of the objective of this work is to find methodologies to assess and minimize the degradation of availability or capacity caused by those factors in various system configurations.

\subsubsection{State graph}

In order to represent the state of the diversity network at a given time, it can be convenient to adopt the graph representation of figure 3. Knowing the initial state composed by the set of active $G W$ at a given time $t_{i}$, denoted $\mathcal{S}_{i}^{j}$, it gives 
the possible sets of active $G W$ at time $t_{i+1} \mathcal{S}_{i+1}^{k}$, after a switching decision (or a non switching one). This graph contains only the transitions between the sets that are possible according to the payload redundancy structure of figure 1 . It is implicitly assumed that the duration of the switching procedure is $\Delta t=t_{i+1}-t_{i}$ and that the decision to switch can be taken at each time $t_{i}$. Between $t_{i}$ and $t_{i+1}$ the active gateways are kept unchanged. If one of this active gateway experience too severe propagation conditions it will results in an unavailability of the feeder link.

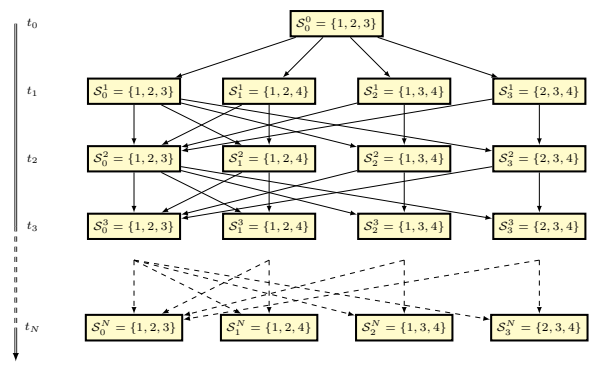

Figure 3: Switching graph for a $\mathrm{N}=3 \mathrm{P}=1$ diversity

With this representation, the optimal switching decision from time $t_{0}$ to $t_{N}$ will be the oriented path that minimizes the overall unavailability or capacity reduction of the system.

\subsubsection{Cost function}

To evaluate which decision is appropriate for the next possible switching time $t_{i}$, it is necessary to define a cost function. In this case the objective metric that needs to be optimized is either the overall capacity or availability. To evaluate the cost of the transition between the states $\mathcal{S}_{i}^{k}$ and $\mathcal{S}_{i+1}^{j}$, the following cost function can be defined:

$$
\Pi\left(\mathcal{S}_{i}^{k}, \mathcal{S}_{i+1}^{j}\right)=f\left(\mathcal{S}_{i+1}^{j}\right)+n . c_{s w}
$$

where $f\left(\mathcal{S}_{i+1}^{j}\right)$ represents the unavailability or loss of capacity induced by the use of the set of gateways of the $S_{i+1}^{j}$ state between $t_{i+1}$ and $t_{i+2} . n$ is the number of switching decisions taken at time $t_{i}$ and $c_{s w}$ is the loss of availability or capacity of the link caused by the switching (due to network congestion, or transmission delay for instance).

More complex metrics penalizing also massive log-off of the users in case of too long communication disruption have been tested (including a penalty of several minutes if the link is lost for more than some tens of seconds). However their inclusion requires a significant increase in computing complexity and the decisions made with this cost functions are similar to the ones made with simpler cost functions. They are therefore not considered in the following of the analysis. 


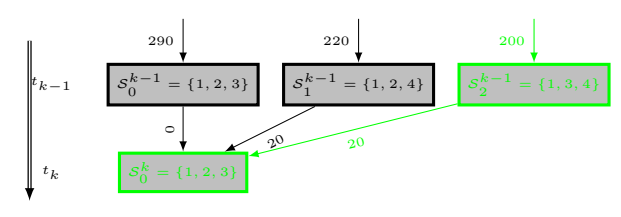

Figure 4: Principle of the iterative search of the optimal path

\subsubsection{Optimization}

If the evaluation of the cost function is perfectly known in the future (there are no prediction errors) the optimal series of decision is the path $\mathcal{P}=\left(p_{0}, p_{1}, \ldots, p_{N}\right)$ that minimizes:

$$
\arg \min _{P} \tilde{\Psi}\left(\mathcal{S}_{0}^{0}, \mathcal{S}_{N}^{p_{N}}\right)=\arg \min _{P}\left(\sum_{i=0}^{N-1} \Psi\left(\mathcal{S}_{i}^{p_{i}}, \mathcal{S}_{i+1}^{p_{i+1}}\right)\right)
$$

The path of Eq. 3 can be computed iteratively as:

$$
\tilde{\Psi}\left(\mathcal{S}_{0}^{0}, \mathcal{S}_{N}^{j}\right)=\min _{k} \tilde{\Psi}\left(\mathcal{S}_{0}^{0}, \mathcal{S}_{N-1}^{k}\right)+\Psi\left(\mathcal{S}_{N-1}^{k}, \mathcal{S}_{N}^{j}\right)
$$

The rationale of the algorithm is illustrated in figure 4. The path of minimum cost to reach each state is computed and stored iteratively from one stage from another.

In practice the state of the channel is not perfectly known in advance, the cost of the state $\mathcal{S}_{i}^{k}$ can not be handled deterministically as it relies on a forecast $\mathcal{F}_{k}^{i}$ of the cost of the occupied state. The optimal decision is in this case the decision path $\mathcal{P}$ that minimizes:

$$
\arg \min _{P} E\left[\tilde{\Psi}\left(\mathcal{S}_{0}^{0}, \mathcal{S}_{N}^{p_{N}}\right) \mid \mathcal{F}\right]=\arg \min _{P}\left(\sum_{i=0}^{N-1} E\left[\Psi\left(\mathcal{S}_{i}^{p_{i}}, \mathcal{S}_{i+1}^{p_{i+1}}\right) \mid \mathcal{F}_{i+1}^{p_{i+1}}\right]\right)
$$

If new and more accurate forecasts are available after the first decision taken in the decision path, it can be updated and the next decisions taken accordingly.

The performances of the operation of smart diversity systems considering various system assumptions, can firstly be evaluated assuming a perfect knowledge of the propagation channel. It results in a upper bound of the performances that can be used to benchmark a real system.

\subsection{Performance evaluation in an idealized case}

In order to assess the impact of various parameters such as the duration of the switching procedure $\Delta t$, or the penalization of the switch $c_{s w}$, synthetic space time correlated time series have been generated to simulate link budgets over gateways spread across Europe with the model described in [?]. For simplification purposes, it is assumed that the link is in outage if the rain attenuation 

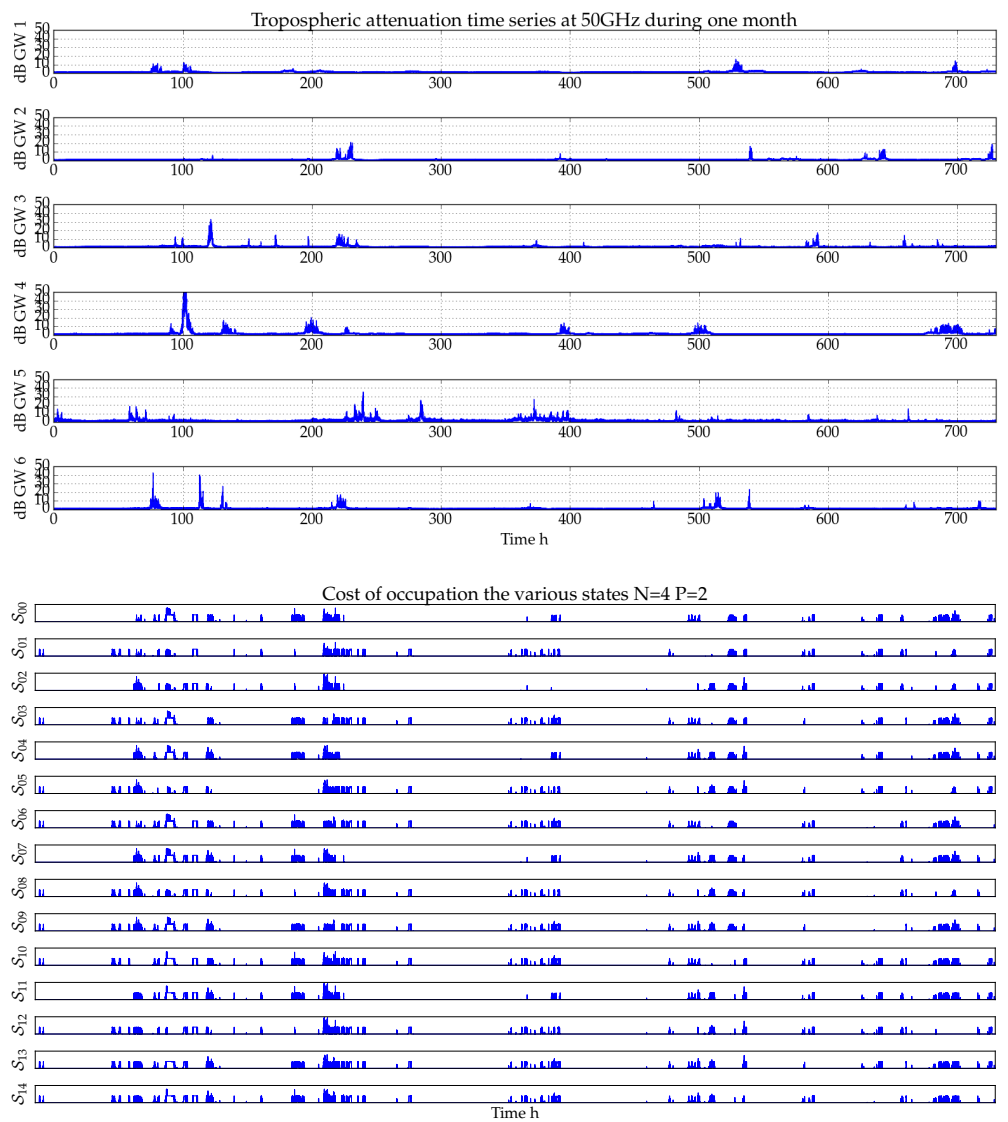

Figure 5: Simulated attenuation time series over 6 sites for one month (top) and concurrent time series of state occupation cost for 5 min intervals for the 12 possible states of a $\mathrm{N}=4 \mathrm{P}=2$ diversity configuration (bottom)

experienced by the various gateways exceeds an attenuation threshold denoted as $A_{t h r}$ in the following.

An example for $N=4$ and $P=2$ is presented with a rain attenuation threshold $A_{t h r}=6 d B$ in figure 5. Other tropospheric effects as gaseous, cloud attenuation are not considered in this study. It is assumed that they can be mitigated through power control mechanisms and that moreover their prediction is less prone to large errors.

From those attenuation time series, the graph optimization methodology described in previous sections has been applied. The cost of the various states has been evaluated according to the availability computed from the time series for the various active GWs combinations. Time intervals spanning from some seconds to one day have been considered for $\Delta t$. It corresponds to the 

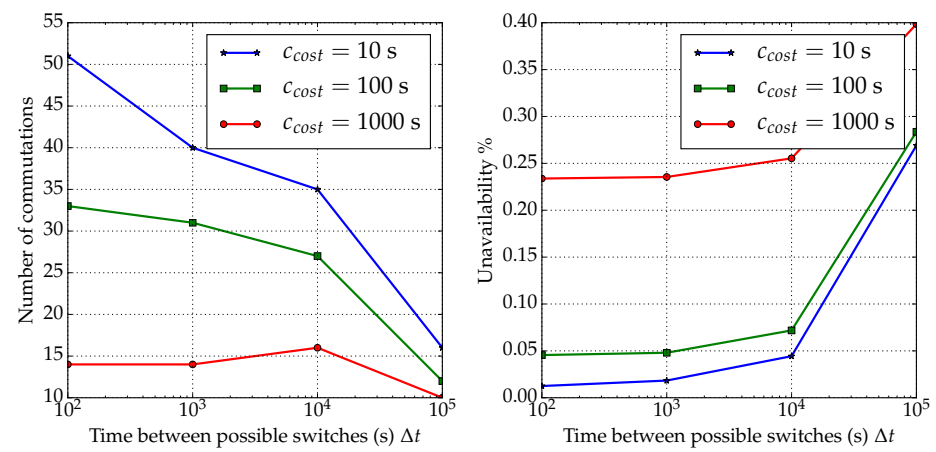

Figure 6: Average number of switches during per month and resulting system availability for a $N=4, P=2$ configuration for various sets of system parameters

extreme cases that range from the possibility to trigger automatically and almost immediately the switch between a nominal and redundant gateway to the possibility to operate the switch once a day in a quasi manual fashion. Various penalizations of the switchings $c_{s w}$ ranging from some seconds to some tens of minutes, depending on the efficiency of the switching mechanisms that have been implemented in the system have also been considered..

The availability resulting from this optimization as well as the number of switches for the various parameters are presented in figure. 6, considering one year of time series.

As what could be anticipated, the time interval between possible switches has a significant impact on the overall availability. For instance with a limited penalization of the switching $c_{s w}$, the unavailability rises from $0.02 \%$ to almost $0.25 \%$ when allowing one switch every $100 \mathrm{~s}$ or one switch per day. The cost of the penalization tends obviously to decrease the number of switches in the optimal path as it is not efficient to switch for too low outage durations. As a counterpart the overall availability is significantly degraded considering the aggregation of the switching cost and of the outage duration of the gateways.

For cost functions based on the optimization of the capacity, similar observations can be made with regards to the interval between successive switches. However, the number of switches will tend to be higher, as low attenuation levels will already cause a decrease of capacity that can be more easily balanced by the loss of capacity caused by the switching between two gateways. The algorithm can be extended to larger network of gateways with a complexity proportional to the number of states to be evaluated, that grows according to the number of combinations of $\mathrm{P}$ among $\mathrm{N}$. 


\section{Propagation channel forecast}

In an operational system, on top of the performance degradation due to the time to perform the switching and to the reduction of capacity caused by the switching, there is also the possibility that the optimal path in a probabilistic sense of Eq. 5, is actually non optimal. The actual cost of the occupied state may differ from the forecasted one. This will happen often if the conditional variance of the cost of occupation of a state knowing the forecast $\operatorname{Var}\left[\mathcal{S}_{i}^{k} \mid \mathcal{F}_{i}^{k}=f\right]$ is high. In simpler terms, it means that sub-optimal switching decisions will be taken often if the forecasts on the state of the channel are inaccurate.

To control the system through the algorithm described at previous section, an evaluation of the outage or capacity reduction caused by propagation on each feeder link during the time interval $\Delta t$ is required. This time interval $\Delta t$ spacing varies between some seconds and some days. There is currently no means to perform efficient forecasts of rain attenuation for this whole range of time horizons. Thus various forecast strategies and data sources have been chosen to this aim:

- for switching rates $\Delta t$ up to 5 minutes, the forecast is based on the current state of the channel.

- for switching rates $\Delta t$ between 5 minutes and 1 hour, the forecast is based on a product named 2PIR issued by Météo-France, that is a temporal extrapolation of rain fields observed by weather radar.

- for switching rates $\Delta t$ comprised between 1 hour and 1 day, data from the high resolution operational weather forecast model of Météo-France named Arome are used.

For those various data sources and parametrization a model of conditional expectation $E\left[\Psi\left(\mathcal{S}_{i}^{p_{i}}, \mathcal{S}_{i+1}^{p_{i+1}}\right) \mid \mathcal{F}_{i+1}^{p_{i+1}}\right]$ is built to estimate the expected cost of occupation of a set of GW during a time interval $\Delta t$, considering the forecast.

This model can be used to determine the optimal path according to Eq. 3. The actual performances can afterwards be assessed with actual propagation data concurrent to the forecast. The capacity and availability of the system can hence be assessed considering the sets of active gateways at each time step, combining the propagation conditions on those gateways and the penalizations induced by the various switches.

\subsection{Available propagation data}

In order to evaluate the performances of the various forecasts methods presented in the remaining of this section, and also to parameterize the models, propagation data collected in the South of France during a joint ONERA-CNES campaign propagation measurement campaign have been used [?]. The location of the various sites over which data have been collected is illustrated in figure 7. 


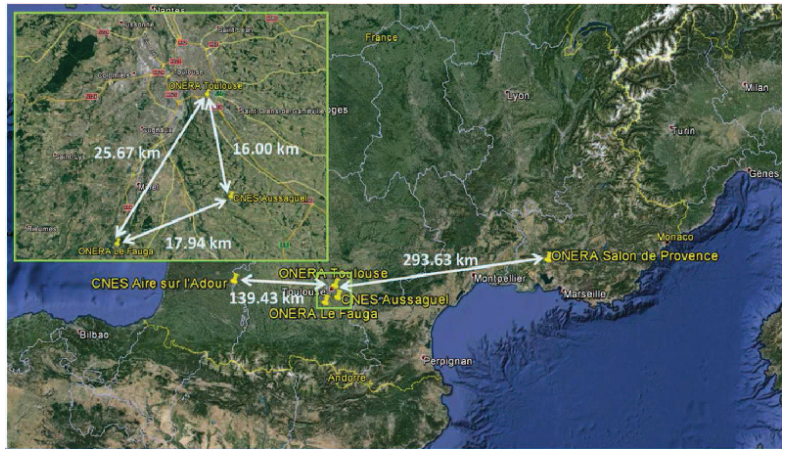

Figure 7: Position of the sites in the South of France where propagation data have been collected simultaneously

Those data have been collected measuring the power fluctuations of Astra $3 \mathrm{~B}$ beacon at $20 \mathrm{GHz}$ with one receiver in the five experimental facilities. Rain attenuation has been separated from the other effects during the processing. To be representative of the performances that could be achieved on a $\mathrm{Q} / \mathrm{V}$ band feeder link. The data have been extrapolated at $50 \mathrm{GHz}$ using ITU-R Rec P.618 long-term frequency scaling model (section 2.2.1.3.2).

\subsection{Forecasts based on the current state of the channel}

For short time horizons, the easiest way to forecast the attenuation is to rely on its current and possibly past estimates. Attenuation estimates at the gateway site can be obtained through beacon measurements or measure of the signal to noise ratio with the analysis of pilot symbols. Knowing this estimate, it is possible to infer the probability distribution of the attenuation in the future. Various models based on Markov chain [?], ARMA models [?] or Kalman filters have been proposed in the literature. One of the simplest and most commonly used model is to consider that the log rain attenuation is a first order Gauss-Markov process (or $\mathrm{AR}(1))[?]$. Under this assumption the pdf of rain attenuation at time $t$ knowing the rain attenuation at time $t-\Delta t$ can be expressed as:

$$
P\left(A(t)>A^{*} \mid A(t-\Delta t)\right)=\frac{1}{2} \operatorname{erfc}\left(\frac{\ln \left(A^{*}\right)-m_{\mid A(t-\Delta t)}}{\sqrt{2} \sigma_{\mid A(t-\Delta t)}}\right)
$$

with:

$$
\begin{aligned}
& m_{\mid A(t-\Delta t)}=m(1-\exp (-\beta \Delta t))+\ln (A(t)) \exp (-\beta \Delta t) \\
& \sigma_{\mid A(t-\Delta t)}=\quad \sigma \sqrt{1-\exp (-2 \beta \Delta t)}
\end{aligned}
$$




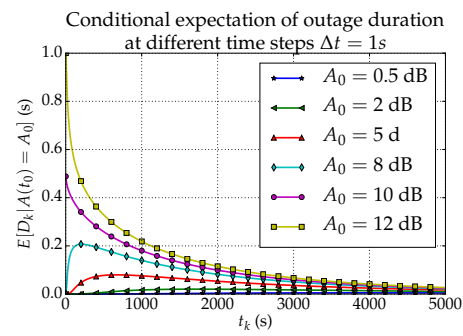

Figure 8: Expectation of the duration spent over $10 \mathrm{~dB}$ of rain attenuation for $1 \mathrm{~s}$ for various time horizons

where $m$ and $\sigma$ are respectively the mean and standard deviation of the $\log$-rain attenuation process and $\beta$ is a correlation parameter usually with a value around $10^{-4} \mathrm{~s}^{-1}$. If the time horizon for which the availability forecast is sufficiently small with regards to $1 / \beta$, it is possible to approximate the duration during which the link has an attenuation over the outage threshold by the probability to exceed the threshold and thus $E(D(t) / \Delta t \mid A(t-\Delta t) \approx P(A(t)>$ $\left.A^{*} \mid A(t-\Delta t)\right)$. The expectation of the duration can then easily be expressed and is represented for arbitrary parameters in figure 8 .

Thus assuming that the cost of the state occupation is the unavailability duration for each gateway, the knowledge of the attenuation for each link at time $t$ allows determining the optimal path through the knowledge of the conditional expectation $E[D(t) \mid A(t-\Delta t]$ and the resolution of Eq. 5. Obviously the quality of the forecast will depend on the parameterization of $\mu, \sigma$ and $\beta$. However, this impact is limited for small forecast horizon as the forecasted attenuation values are strongly correlated with the current ones. It is also possible to include in the optimization process the estimation noise to account for attenuation measurement uncertainty.

This control technique will be shown to give good results for short prediction horizon. Nevertheless as illustrated in figure 8, the correlation decays relatively quickly and after some thousands of seconds the expectation to exceed the threshold is almost the same disregarding the initial attenuation value. It implies that the conditional variance is important and that the forecast becomes inaccurate. Thus for longer time horizons, other means to operate the diversity system have to be found.

\subsection{Forecasts based on extrapolated radar data}

Rain fields are in most of European countries continuously scanned by weather radar networks that constitutes a valuable source of information for the monitoring of extreme events like flash floods or to provide inputs to hydrology models. For flood forecast purposes mechanisms to extrapolate the rain reflectivity from current observation (displacing the various features according the local advection vector) have been developed [?]. A product named 2PIR implementing this 


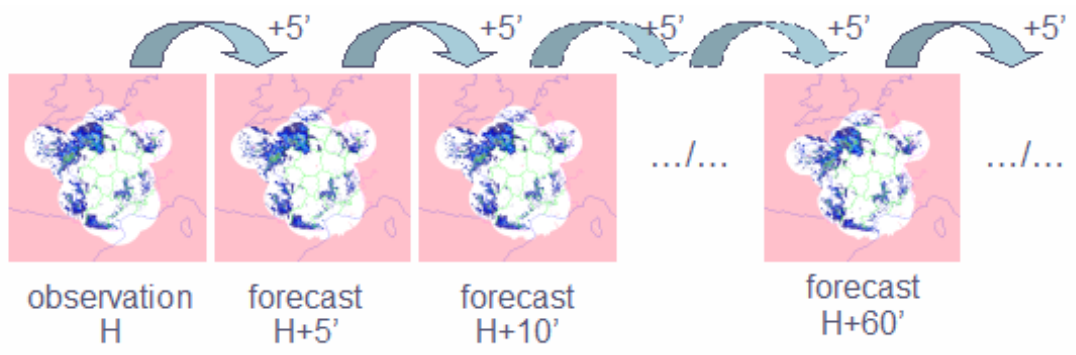

Figure 9: Example of 2PIR products forecasts based on an extrapolation of weather radar data
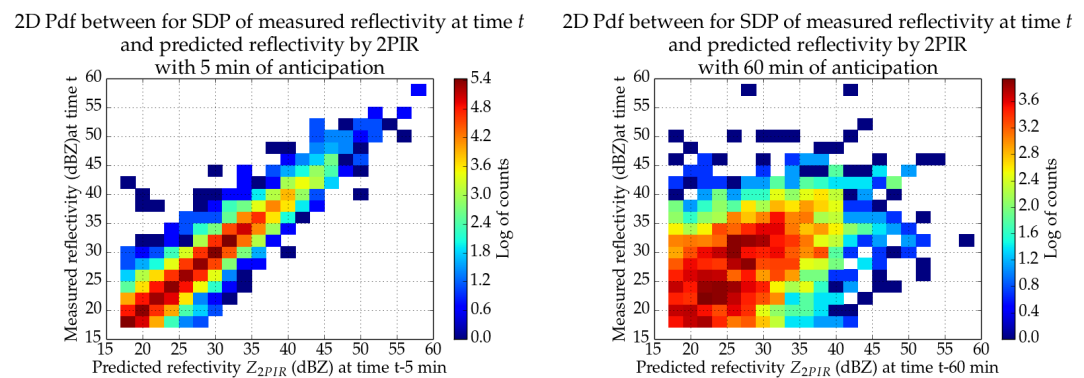

Figure 10: 2D histogram of measured reflectivity function of forecasted reflectivity for a forecast 5 minutes in the future (left) and a forecast one hour in the future (right)

algorithm is released operationally by Météo-France (over France) and has been used for this study. Similar products are also issued by meteorological offices in areas covered by weather radar networks. As illustrated in figure 9 forecasts of reflectivity between 5 an 60 minutes in the future are produced by $2 \mathrm{PIR}$. The grid spacing of those forecasts is of $1 \mathrm{~km}$ and they are refreshed each 5 minutes after each update of the radar measurements.

The reflectivity measured by radar is linked to the concentration of raindrops in the atmosphere. This concentration of raindrops can also be linked to the specific attenuation undergone by a microwave link. Therefore, it seems plausible to expect a link between the forecasted radar reflectivity and rain attenuation. In order to find this link and build a model relating the fraction of a time interval $D(t)$ for which the link has an attenuation above a given level of attenuation $A_{t h r}$ function of the reflectivity $Z_{2 P I R}(t-\phi)$ forecasted from the reflectivity field measured at time $t-\phi$, a two step decomposition has been used. The first step has been to relate the forecasted reflectivity to the reflectivity actually measured $Z_{\text {meas }}$ at time $t$ and to determine the conditional probability distribution $P\left(Z_{\text {meas }}(t) \mid Z_{2 P I R}(t-\phi)\right)$ that is illustrated in figure 10 for different time horizons. 


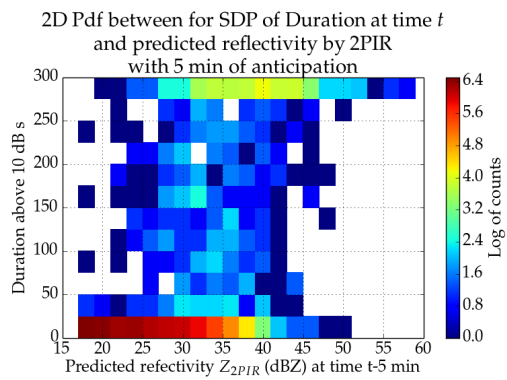

Figure 11: 2D histogram of measured reflectivity and duration within a $5 \mathrm{~min}$ interval over a $10 \mathrm{~dB}$ rain attenuation threshold

The second step is to relate the fraction of the time interval $D(t)$ for which the link is above a given attenuation threshold $A_{t h r}$ to the measured radar reflectivity at time $t$ and to determine the conditional probability distribution $P\left(D(t) \mid Z_{\text {meas }(t)}\right)$. The characteristics of this parametric predictor is illustrated in figure 11.

Parametric models depending on the climatic features of each sites have been derived for both probability distributions. Those two probability distributions can be combined to get the probability distribution of the fraction of the time during which the link is above the threshold during a time interval $\Delta t$ knowing the reflectivity forecast $Z_{2 P I R}$ made at time $t-\phi$ through:

$$
P\left(D(t) \mid Z_{2 P I R}(t-\phi)\right)=\int P\left(D(t) \mid Z_{\text {meas }}(t)\right) P\left(Z_{\text {meas }}(t) \mid Z_{2 P I R}(t-\phi)\right) d Z_{\text {meas }}
$$

It is then straightforward to deduce the conditional expectation $E\left[D(t) \mid Z_{2 P I R}(t-\phi)\right]$ from Eq. 7. The value of this conditional expectation is illustrated in figure 12 for various time horizon of the forecasted reflectivity.

Unsurprisingly, the expectation of outage duration decreases with the increase of the threshold whatever the forecasted reflectivity. Also due to the tighter link between measured and forecasted reflectivities for short forecast times, the selectivity of the conditional expectation is higher for short forecast time than for forecast times approaching one hour. For longer time lags another type of forecast has to be used.

\subsection{Forecast based on numerical weather prediction}

For prediction of rain attenuation at longer time scales, the prediction of attenuation from rain field extrapolated by radar is no longer efficient as the forecasted and measured reflectivities becomes less and less correlated as shown in figure 10. Thus, means to provide forecasts on rain related variables for longer durations have to be found. This can be achieved through the use of numerical 

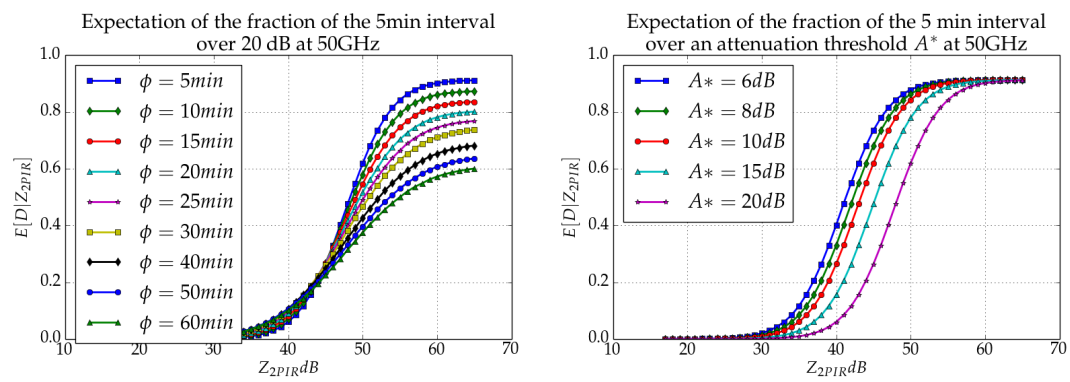

Figure 12: (left) Conditional expectation of the fraction of the duration $D(t)$ spent above $10 \mathrm{~dB}$ of attenuation function of the reflectivity forecast for various ages $\phi$ of the reflectivity forecast $Z_{2 P I R}(t-\phi)$. (right) Conditional expectation of the fraction of the duration $D(t)$ spent above various attenuation threshold $A^{*}$ function of the reflectivity forecast aged from 5 minutes

weather forecasts. More specifically in this study, data from AROME, the numerical weather forecast model used by Météo France, have been used [?] . The outputs of interest of the model are hourly rain amounts with a spatial resolution of $2.5 \mathrm{~km}$. Forecasts for time horizons ranging from $3 \mathrm{~h}$ to $36 \mathrm{~h}$ after its initialization are available and the computations are refreshed on a $6 \mathrm{~h}$ basis.

The model for the expectation of outage in a given state knowing the forecast has been established as what has been done for extrapolated reflectivity fields to split the expression of the conditional distribution into two terms. One term depicts the dependence between the rain forecast $R_{A r}(t-\phi)$ and observed rain amounts $R_{A n t}(t)$ from gridded data of a raingauge network (named Antilope). The other term is quantifying the link between the rain amount $R_{A n t}(t)$ observation and the fraction of the duration $D(t)$ spent in a given state that lies over a given attenuation threshold. The distribution $P\left(R_{A n t}(t) \mid R_{A r}(t-\phi)\right)$ is illustrated in figure 13 for a time horizon of $9 \mathrm{~h}$ for the forecast. In fact due to the very low correlation between observed and forecasted rain amounts as shown in figure 13, only two classes of forecast to avoid a dramatic increase of false alarms are considered. The two classes considered are predicted hourly rain amount below $0.1 \mathrm{~mm}$ or over $0.1 \mathrm{~mm}$.

The distribution $P\left(D(t) \mid R_{A n t}(t)\right)$ is illustrated in figure 14 where there also, the dispersion between the two quantities appears to be high. The development of parametric models for those two distributions enables to get a model for the conditional probability $P\left(D(t) \mid R_{A r}(t-\phi)\right)$ through:

$$
P\left(D(t) \mid R_{A r}(t-\phi)\right)=\int P\left(D(t) \mid R_{A n t}(t)\right) P\left(R_{A n t}(t) \mid R_{A r}(t-\phi)\right) d R_{A n t}
$$

The conditional probability $P\left(D(t) \mid R_{A r}(t-\phi)\right.$ can then be used to deduce the conditional expectation and to determine the path in the switching graph if the minimum time between the switches exceeds an hour. 


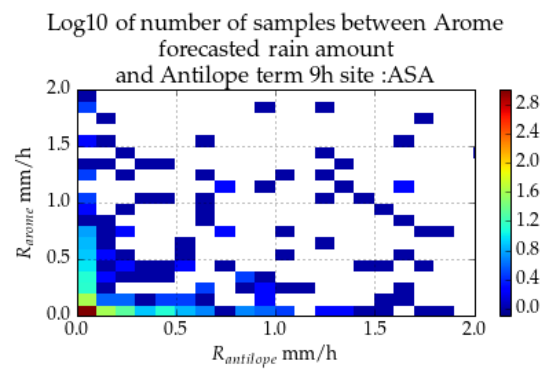

Figure 13: 2D histogram comparing rain amount estimated from gridded raingauge networks and from rain amount forecasted with a time horizon of $9 \mathrm{~h}$

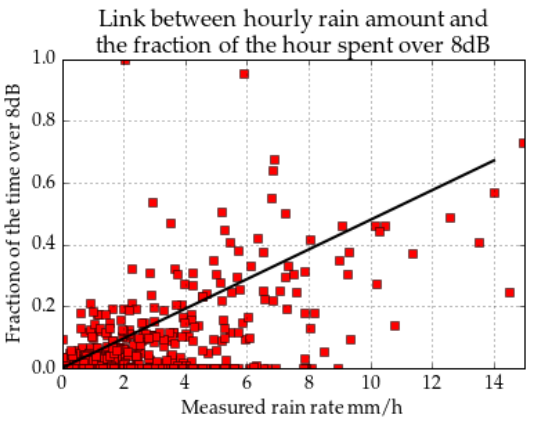

Figure 14: Scatter plot between measured rain amount $R_{a n t}(t)$ and fraction of an hour $D(t)$ spent over $8 \mathrm{~dB}$ 


\section{Performance evaluation}

In order to evaluate the performances of the various solutions developed, forecast data concurrent to the collected propagation data have been considered. The different algorithms have been tested using concurrent inputs, corresponding to the period of propagation data acquisition.

\subsection{Examples of switching decision time-lines}

For the various prediction algorithms input data have been provided for one month (August 2014) and the optimal path computed according to Eq. 3. It yields to the results of figure 15. For the left columns, the horizontal axis is the time, the vertical axis is the forecast horizon starting from the time in abscissa and the color code is the evaluation of the cost of state occupation. The right column presents the forecasted activity of the various ground stations. The ground station of the bottom acts as the redundant one. The state actually used corresponds to the bottom line of each graph of the right column. If there were a disruption of the data flow, the decision based on older forecasts could be taken.

The forecast of gateway usage are illustrated on figure 15 for one month (the same for all algorithms), the forecast time is limited to one hour for methods based on extrapolated weather radar data and SNR whereas it is up to 1 day for numerical weather forecast based method.

\subsection{Performance assessment}

This analysis has been extended to the complete period ( 5 months) during which data where available for the five experimental sites.

Generalizing the analysis to a longer period ( 5 months) and comparing with the actual availability obtained using the measured propagation data yields to the results of table 1 . The case "channel perfectly known and possibility to commute costlessly and instantaneously" corresponds to the case for which the availability can be evaluated through combinatorial analysis. The case "channel perfectly" known with 5 min of delay between the triggering of the switch and the actual switch could correspond to the performances that can be reached on a real system with an ideal forecast of the channel state. In this case, it can already be noticed that the unavailability is significantly degraded with regards to the first case. The SNR and radar rows corresponds to the performances that could be obtained controlling the system either through a monitoring of the instantaneous attenuation at each gateway site or with the extrapolation of rain reflectivity observed by weather radar. The combining of those two methodologies (by combining the cost functions in the optimisation) do not improve significally the results but more efficient control methodology may be found with further investigations. In this case the unavailability is also increased in a significant proportion by a factor 2 and 3. Lastly, assuming switching possibilities every hour, without prediction errors, the unavailability is increased 

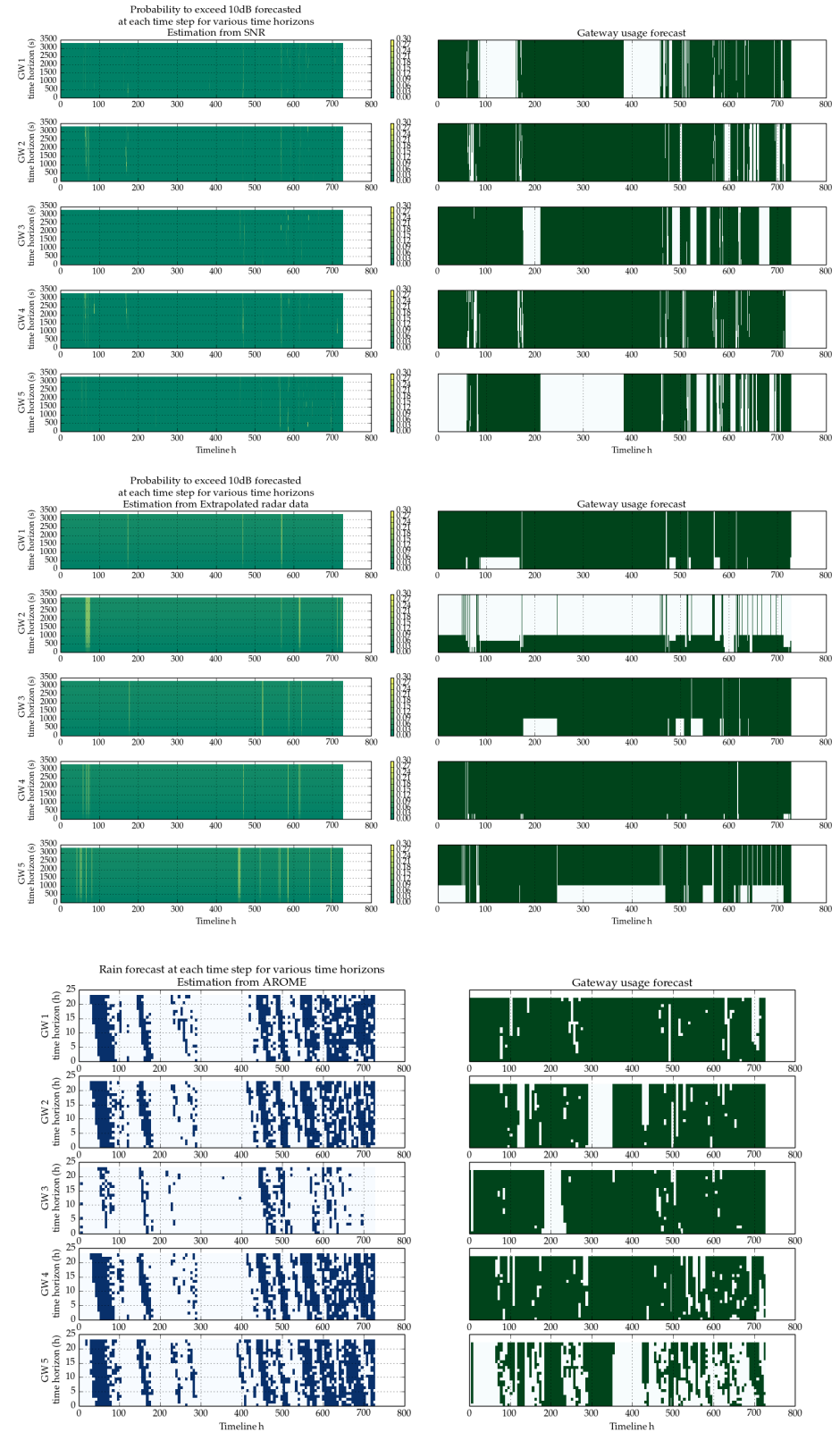

Figure 15: Example of cost function evaluation for one month for a $\mathrm{N}=4, \mathrm{P}=1$ diversity configuration, using SNR forecast algorithm, Extrapolated radar based algorithm (2PIR), NWF based (AROME) algorithm 


\begin{tabular}{|l|l|l|l|l|}
\hline Forecast method & $\begin{array}{l}\text { Min time between } \\
\text { switches }\end{array}$ & Cost of a switch & Number of switches & Unavailability \% \\
\hline \hline Ideal control & $0 \mathrm{~s}$ & $0 \mathrm{~s}$ & 450 & $1.110^{-3} \%$ \\
\hline Ideal control & $300 \mathrm{~s}$ & $2 \mathrm{~s}$ & 362 & $2.310^{-3} \%$ \\
SNR based control & $300 \mathrm{~s}$ & $2 \mathrm{~s}$ & 1252 & $3.9-3 \%$ \\
$\begin{array}{l}\text { Extrapolated radar based } \\
\text { control }\end{array}$ & $2 \mathrm{~s}$ & 1413 & $5.610^{-3} \%$ \\
$\begin{array}{l}\text { Combined SNR/radar con- } \\
\text { trol }\end{array}$ & $300 \mathrm{~s}$ & $2 \mathrm{~s}$ & 1513 & $4.110^{-3}$ \\
\hline Ideal control & $10 \mathrm{~s}$ & 115 & $3.610^{-2} \%$ \\
NWF based control & $3600 \mathrm{~s}$ & $10 \mathrm{~s}$ & $1.710^{-1} \%$ \\
\hline \hline
\end{tabular}

Table 1: Summary of the performances of the various algorithms evaluated with measured propagation data, $\mathrm{N}=4 \mathrm{P}=1$, outage threshold $12 \mathrm{~dB}$ at $50 \mathrm{GHz}$ for rain attenuation (based on the 5 months of data fully available)

again by one order of magnitude, while it is more than two if the switches are controlled with the numerical weather forecast data.

\section{Conclusion}

This paper has described a control methodology to operate a network of gateways in $N+P$ configuration, for a wide range of parameters in terms of maximum switching rate, and loss of capacity or availability induced by the switching. In order to assess the changes of availability with regards to a simple combinatorial analysis, the system has been modelled as a graph crossing problem. The optimal control is achieved by minimising a cost function associated to the graph crossing. Performance evaluations have been conducted firstly assuming a perfect knowledge of the propagation channel to define a performance upper bound. Then the performances have been evaluated using various forecast data sources, appropriate for different time horizons. From short to long time horizons, those forecasts can be based on an extrapolation of the instantaneous attenuation experienced by the feeder link, on the extrapolation of radar data and also on numerical weather prediction outputs. The various models to perform the cost function forecast have also been detailed. Those models have been developped to be parametric with regards to the feeder link sites climatology.

In terms of overall performance it has been shown that the increase of the minimum time between two switchings greatly degrades the performances as it may increase the unavailability by some orders of magnitude. It may require to be accounted for at the design stage. Otherwise, there is a risk to deviate too significantly from the initial availability specifications when designing the system. The control of the switches based on attenuation measurements seems to offer fair performances for low time latencies. Extrapolated radar data could be also a viable alternative that do not degrades too significantly the performance for latencies of some tens of minutes. However those products should be available over all the gateways. It will potentially require the support from several meteorological offices. The use of numerical weather forecasts to control the diversity seems dangerous due to the too low level of instantaneous correlation with the data. It could however be a good tool to schedule maintenance on 
the systems. Improvement on the control of the system may also be brought by the use of probabilistic forecasts, that could help the evaluation of the costs by associating a plausibility to the various potential meteorological scenarios. It will also be necessary to forecast the other propagation impairments like cloud and gaseous attenuation. Nevertheless, considering their lower variability and higher magnitude, this could be more easily addressed with meteorological data.

A consolidation of the different parameters proposed in this study can be undertaken on a larger scale using $\mathrm{Q} / \mathrm{V}$ band data acquired from Alphasat payload in various sites in Europe. Among the other prospects of this work, the application of a similar formalism to control the switching for an optical feeder link could be of interest. In this case the problematic will be to forecast the cloudiness or optical depth above the various feeder links.

\section{Acknowledgment}

The authors would like to thank CNES for funding the activity in the framework of the contract "CNES R-S13/TC-0005-043". 